\title{
Requirements Engineering and the Creative Process in the Video Game Industry
}

\author{
David Callele, Eric Neufeld, Kevin Schneider \\ Department of Computer Science \\ University of Saskatchewan \\ Saskatoon, Saskatchewan, Canada S7N 5C9 \\ \{callele,neufeld,kas\}@cs.usask.ca
}

\begin{abstract}
The software engineering process in video game development is not clearly understood, hindering the development of reliable practices and processes for this field. An investigation of factors leading to success or failure in video game development suggests that many failures can be traced to problems with the transition from preproduction to production. Three examples, drawn from real video games, illustrate specific problems: 1) how to transform documentation from its preproduction form to a form that can be used as a basis for production, 2) how to identify implied information in preproduction documents, and 3) how to apply domain knowledge without hindering the creative process. We identify 3 levels of implication and show that there is a strong correlation between experience and the ability to identify issues at each level.

The accumulated evidence clearly identifies the need to extend traditional requirements engineering techniques to support the creative process in video game development.

Keywords: Non-functional requirements, elicitation, video game development, game design document, preproduction, production, domain-specific terminology.
\end{abstract}

\section{Introduction}

Video games are a special type of multimedia application - an entertainment product that requires active participation by the user. Developed by a multi-disciplinary team, non-functional requirements such as entertaining the user creates special demands on the requirements engineering process. Requirements like fun and absorbing are not well understood from the perspective of requirements engineering, compounding communication issues between game designers and software engineers. Game designers may not understand, for example, the limitations of artificial intelligence when designing non-player characters while software engineers may not understand the creative vision or they may be too willing to compromise that vision in the rush to ship the product.

It may be that nothing can qualitatively change this. However, it should be possible to decrease the cost of delays caused by communication errors in such a nonheterogeneous group. As a first step toward the development of a formal process, we have tried to locate the causes of the most costly errors. By way of background, we first review the requirements engineering literature applied to multimedia development and introduce the video game industry and the video game development process, with attention to the roles of preproduction and the game design document (as a deliverable artifact of the preproduction process). We analyze the observational reports from the Postmortem column in Game Developer magazine, categorize the information therein, and present the results. Three examples, drawn from real video games, illustrate particular issues that must be addressed in a formal process. We follow with our conclusions and directions for future work.

\section{Background}

Requirements engineering within a community of common interest is difficult - the ability to precisely communicate and capture stakeholder wants and needs is rare. Traditional requirements engineering techniques [12] [22] assume these communications issues can be overcome in a few iterations. However, we are unaware of any work that directly addresses the validity of this assumption in a multi-disciplinary development effort. When these efforts include a strong creative or artistic element, such as in video game design, multimedia web sites, or the movie industry, even the industrial literature is sparse.

Members of video game development teams include practitioners from such diverse backgrounds as art, music, graphics, human factors, psychology, computer science, and engineering. Individuals who, in other circumstances, would be unlikely to interact with each other on a profes- 
sional basis unite in their economic goal of creating a commercially successful product. Requirements engineering in the face of such diversity requires the creation of a common (domain) language (and implied world model) specific to the task at hand. Once all stakeholders fully commit to the domain language, then a set of requirements that captures the stakeholders wants and needs can be generated.

Given the dearth of directly related work, we performed a more extensive literature review, focussing on: 1) requirements engineering and emotional factors (including fun in games), 2) issues of language and the creation of a common language or domain ontology, 3) requirements elicitation and the effects of feedback on emergent requirements, particularly in multimedia development, and 4) the roles of implication, inference, and anticipation when capturing requirements.

\subsection{Emotional Factors}

Few researchers have investigated emotional factors in requirements engineering. Draper [8] looked at fun as a candidate software requirement, attempting to identify what it is that makes play "fun". He concluded that "fun is not a property of software, but a relationship between the software and the users goals at that moment" and that "providing enjoyment is now a defining requirement of an important class of software, and this has not been sufficiently recognized in our analyses and design methods". These conclusions are consistent with our experience.

Hassenzahl et al. [14] introduced hedonic qualities (those that are unrelated to the current task but present for emotional reasons) and associated repertory grid techniques for measuring them. Bentley et al. [3] investigated emotional (affective) factors in computer games, noting that "software requirements for these and other affective factors are never truly captured in an official manner". In particular, usability, flow (immersion), and motivation were considered via a user survey mechanism. They note that there are no established techniques for eliciting emotional requirements. Even Chung, in his detailed analysis of non-functional requirements [7], does not substantively address emotional issues.

In the field of game design, Salen and Zimmerman [24], Laramee [16], and Saltzzman [25] address issues of emotions and emotional response in game players. While these works do not directly address requirements engineering practices, the techniques that they describe for game design and eliciting feedback from players may increase the range of elicitation techniques available to practitioners. In a more general sense, Norman [20] describes numerous human factors practices that could be readily incorporated into requirements engineering for video games.

\subsection{Language and Ontology}

Zave [28] classifies the problems addressed by requirements engineering, defining the domain, in part, as "...translation from informal observations of the real world to mathematical specification languages." In game development, this is only partially true. In many cases, the game designer, an individual who may have little or no interest in a mathematical representation, is also tasked with generating the requirements. Unable to generate the requirements in isolation, the game designer works with the production team to translate the vision to requirements - usually stated in natural language complete with domain specific terminology. Once captured, the requirements may be formalized in place or, more likely, formalized as they are translated into specifications.

Goguen [13] describes creating a common language as the creation of discourse structures that "... are situated, emergent, open, locally organized, and contingent." In other words, the domain language must be taken in context, as created by the members of the domain, constantly subject to revision, affected by current developments, and subject to re-interpretation in light of historical and anticipated events. The dynamic nature of this description applies well to the evolutionary aspects of video game development.

Jarke and Pohl [15] describe a central system vision, driven by a perceived need for change (either threat or opportunity). They further stress the importance of the vision, defining "requirements engineering as a process of establishing vision in context". An accurate expression of the "vision in context" requires a language of discourse that includes the requisite domain specific terminology. Van Lamsweerde [27] describes this domain specific terminology as the "conceptual units in terms of which models will be built".

Pinheiro [21] introduces requirements honesty, maintaining the integrity of the requirements engineering process in the face of significant time-to-market pressures (such as those experienced in the video game industry). Process integrity breakdown may be reflected in the domain language - when domain language becomes imprecise, this may be an indicator of trouble.

The need for an ontology (a formal representation of the entities within a domain and their relationships) is mentioned [15], [27] but the focus of the work seems to be on business process improvements rather than new product development. Ontologies themselves have be considered a deliverable from the requirements engineering process [6].

Automated approaches to requirements capture have been tried, particularly in the area of translating natural language to a formal representation. Gervasi and Neusebeih [11] proposed a lightweight approach, and in their case study were able to identify errors missed by other valida- 
tion methods. Their approach relied heavily on a restricted domain of discourse and was focussed on the validation of objects and actions in this environment. While their syntactic analysis has significant benefit, the complexity of the problem in a (relatively) unrestricted domain such as video game design may exceed the bounds of practicality. Results in this area would help the documentation transition studied further in Section 6.1

\subsection{Elicitation, Feedback and Emergence}

Goguen [13] emphasizes that "feedback and feedforward go on all the time, at least in successful large projects" and that "requirements are emergent". Emergent requirements, those that are discovered during the transition from preproduction to production, are a significant aspect of the creative design process.

Sutton [26] points out that it is only through an iterative process that we can hope to achieve an acceptable degree of certainty when capturing requirements. Interactions between language of discourse and abstraction heighten the complexity.

Using web based artifacts as an example of multimedia development, Lowe [17] characterizes development of web-based systems by "uncertainty in the project domain and volatility of the client needs and available technology." concluding that "Research has tended to focus on design methods, but largely without considering how these design processes contribute to a domain understanding and clarification of the requirements." The volatility and lack of clarity described here is also characteristic of video game development.

Zave [28] presents a classification scheme that assumes that "... as software engineers, we can seek to understand social factors but we can only hope to influence technical practices." We posit that requirements engineering can take a more proactive approach in video game development by providing feedback from production to preproduction in response to a feedforward of early versions of preproduction documentation. The resultant influence on the creative process escapes Zave's technical practices restriction. Specific feedforward and feedback examples appear in Section 6.3

\subsection{Implication, Inference and Anticipation}

In practice, requirements elicitation consists of repeatedly asking what and why of the customer. As Van Lamsweerde [27] states, further goals are elicited through questioning why a current goal exists or is in its current form.

However, the customer is not always consciously aware of all of the goals. The search for implied goals may be the most important duty of a requirements engineer. So- phisticated practitioners look for implications as soon as possible and present them to the customer to evaluate their validity. Van Lamsweerde [27] notes that "The lack of anticipation of exceptional behaviors may result in unrealistic, unachievable and/or incomplete requirements."

The role of implication in emergent requirements is investigated further in Section 6.2.

\section{Video Game Development}

Video games are a significant element of the entertainment industry. The Consumer Electronics Association [1] reports that entertainment software sales rose from $\$ 5.1$ billion in 1999 to $\$ 7.7$ billion in 2003 and that hardware sales increased from \$2.3 billion in 1999 to \$3.2 billion in 2003 . Combined hardware and software sales in the video game industry exceed the $2003 \$ 9.42$ billion gate receipts of theatrical release movies in North America [10].

However, for every advertisement for a newly released game, the trade press reports a disproportionately large number of projects that fail to reach the market. The present work begins an investigation into the causes of these failures. The multidisciplinary nature of the video game development process - with art, sound, gameplay, control systems, human factors (and many others) interacting with traditional software development creates complexities that may recommend a specialized software engineering methodology for this domain.

\subsection{Development Process}

Figure 1 models the game development process as two consecutive efforts. The left hand side of the diagram depicts the preproduction phase, resulting in a Game Design Document (GDD). Preproduction loosely corresponds to a customer's internal efforts to define their wants and needs before meeting with the development team.

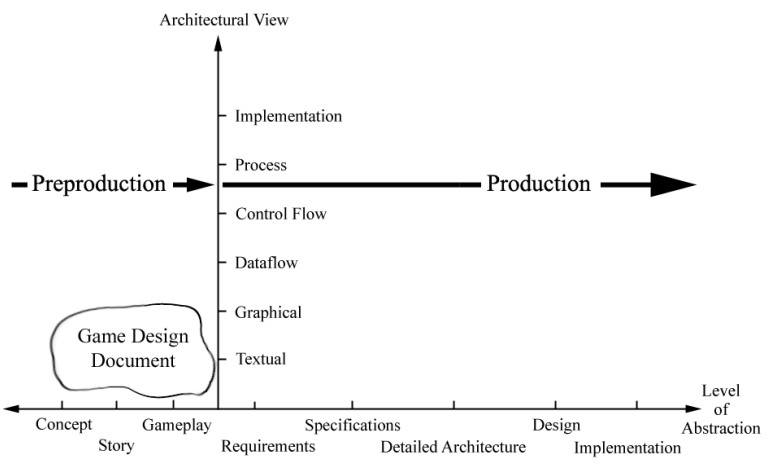

Figure 1. Video game development 
The right hand side of the diagram, derived from Medvidovich and Rosenblum [18], depicts the production phase. During the production phase, the GDD is analyzed (with the assistance of the game designer(s)) to capture system requirements that are then restated as a formal specification. Once the specification is complete, a traditional software development process begins (often using an iterative development effort of some form), resulting in the game artifact.

Moving from preproduction to production is particularly difficult in video game development. A wide range of factors (e.g. artistic, emotive, and immersive factors) must be addressed by the requirements engineering effort. These factors are captured in the game design document.

\subsection{The Game Design Document}

The game design document is a creative work written by the game designer (or game design team). The GDD must be thorough, but not necessarily formal (in the sense of structure or from a mathematical perspective). In fact, one could argue that imposing too much structure on the creative process may be highly detrimental - constraining expression, reducing creativity, and impairing the intangibles that create an enjoyable experience for the customer. In a sense, the GDD is the requirements document as defined by the preproduction team.

The form of the game design document varies widely across genres and studios. Typically, a GDD (drawing loosely from Bethke [4]) includes a concept statement and tagline, the genre of the game, the story behind the game, the characters within the game, and the character dialogue. It will also include descriptions of how the game is played, the look, feel, and sound of the game, the levels or missions, the cutscenes (short animated movie clips), puzzles, animations, special effects, and other elements as required.

A game design document is a preproduction artifact designed to capture a creative vision. It is not designed to meet the needs of a production effort. If a GDD is being used as a source document in the production phase, there are two possible explanations. The first explanation may be that the game design document contains the information required for the production phase. In this case, the game design document is malformed and should be restructured and maintained as independent preproduction and production documents. The second explanation may be that, even though the game design document does not contain production information, the production team is performing requirements engineering, specification, and possibly even design, on an ad hoc basis. The greatest danger associated with such ad hoc activities is the dependence on human memory for capturing decisions and their justifications.

There are issues associated with managing the game de- sign document to requirements document transition. Two sets of documentation must be created and maintained. The writing styles associated with the two sets of documentation are very different - is it reasonable to expect that a single individual can perform both tasks in an efficient and acceptable manner, particularly in the absence of generally accepted practices for performing this translation?

\section{The Transition from Preproduction to Pro- duction}

Requirements errors are some of the most costly to fix; Boehm and Basili [5] estimate that errors of this type can cost up to 100 times more to fix after delivery than if caught at the start of the project. Despite the available evidence and accumulated experience, many projects still suffer from failures due to inadequate requirements engineering

Game designer and producer Eric Bethke [4] states

...too many projects violate their preproduction phases and move straight to production. ... In my opinion, preproduction is the most important stage of the project. I would like to see the day when a project spends a full 25 to $40 \%$ of its overall prerelease time in preproduction. During production there should to be relatively few surprises.

He promotes the use of UML based tools as a way to manage the transition but a formal (or semi-formal) transition process is not presented. Many of the requisite elements for production management (such as requirements capture, requirement analysis, task analysis, time estimation, project plans and technical design) are discussed in an informal manner.

Other producers and consultants, such as Rollings [23] and Michael [19], also identify many of the requisite elements for production management but do not provide formal or semiformal guidelines for managing the transition.

When discussing game design documents, Bethke [4] states "... I have never seen a completed design document, and one of the reasons is that game design documents need to be maintained through the course of production." With time-to-market pressures so prevalent, it is easy to see how documentation maintenance is given low priority.

Despite the recognized need, we have discovered no evidence that a process for managing the transition from preproduction to production has been proposed (recognizing that such a process may exist within an organization but remain unreported in the literature). 


\section{Review of Postmortem Columns}

The video game industry is competitive and management processes are significant corporate assets and generally inaccessible to the researcher. Therefore, we use the Postmortems columns in Game Developer magazine [2], some of which are extracted in POSTMORTEMS from Game Developer [9], as a source of observational reports on this issue.

From the author's guide provided by the publisher:

... Explain what 5 goals, features or aspects of the project went off without a hitch or better than planned. ... Explain what 5 goals, features or aspects of the project were problematic or failed completely. ... Important: try to come up with things that went right/wrong during project that are likely unique to your project. Stay away from common and well understood problems and solutions (e.g., "communication between the team members wasn't good" - that's been true of most games), and focus on what made your project different from others.

The reports presented in the Postmortem column potentially capture what makes video game development unique. They are typically attributed to members of the project management team or middle to upper management within the development organization. As such, one can reasonably assume that the reports reflect issues of particular import to the authors. While there may be an observer effect, particularly with respect to those items that went wrong, we assume the information presented has a strong basis in fact.

Fifty postmortem reports [2], published between May 1999 and June 2004, were analyzed in an attempt to identify factors that lead to success or failure in video game development. Each report contained 5 entries in the "what went right" and "what went wrong" sections. These entries were reviewed and classified according to the following scheme ${ }^{1}$.

The classifications scheme has five categories: 1) preproduction, issues outside of the traditional software development process such as inadequate game design or inadequate storyboarding, 2) internal, issues related to project management and personnel, 3) external, issues outside of the control of the development team such as changes in the marketplace and financial conditions, 4) technology, issues related to the creation or adoption of new technologies, and 5) schedule, issues related to time estimates and overruns. Schedule issues are a subset of internal issues, but were

\footnotetext{
${ }^{1}$ In an attempt to reduce possible biases, the entries were reviewed with minimal identifying information and categorization of the "what went right" entries was performed independently of the "what went wrong" entries.
}

uniquely identified in an effort to determine if scheduling was a significant issue. Any pair of the five categories was also possible (e.g., "internal and technology") if the entry was that precise.

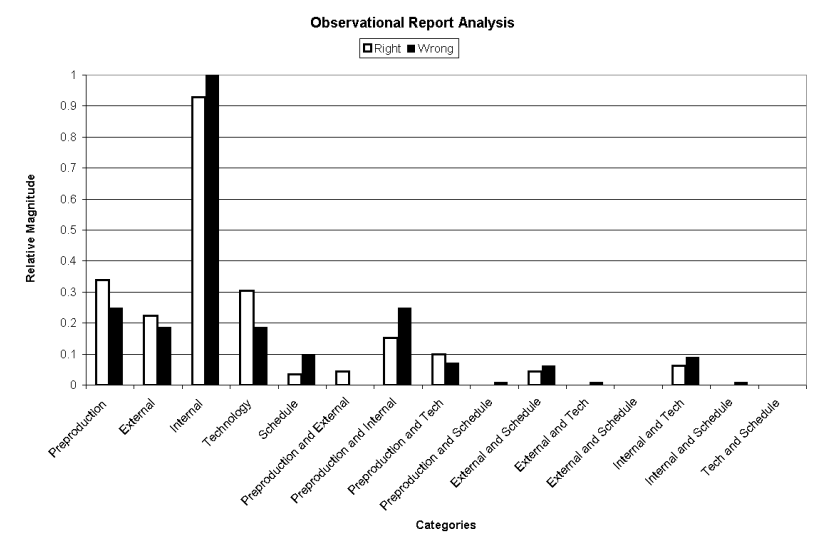

Figure 2. Observational Report Analysis

Figure 2 is a normalized representation of the results of the categorization process. Of the 15 possible categories, only five categories are significant. In order of significance: internal factors, preproduction factors, technology factors, interactions between preproduction and internal factors, and external factors. In relative terms, internal factors dominate any other category by a factor of approximately $300 \%$.

Closer inspection of points classified as internal or schedule factors reveals that many, if not most, of the entries are related to classic project management issues. In particular, missing project elements (tasks) or errors in estimating the size of the tasks are particularly common. It appears that many of these issues could be addressed by a $\mathrm{RE}$ process that better manages the transition from preproduction to production.

Of interest is the balance in the categorization results. Across all categories, across all projects, the maximum deviation from the mean is only $5.8 \%$ - a category was perceived as likely to contribute to the success of a project as it was to the failure of the project.

The high degree of correlation between the "what went right" and "what went wrong" entries could be a result of the granularity of the categorization scheme - approximately $60 \%$ of all entries are categorized within the (major) internal category or related minor categories. In general, the management of different aspects of the production process was often listed both as an element that went right and an element that went wrong within a given project.

The degree of correlation between the "what went right" and "what went wrong" entries within a given project is also significant. We assumed that the order in which the en- 


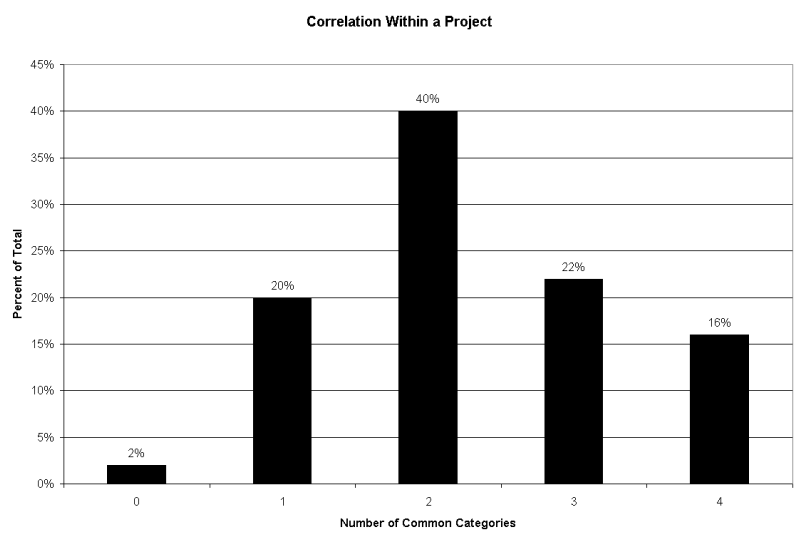

Figure 3. Correlation Within a Project

tries were presented was irrelevant and then cross-checked the results of the categorization process to see if the same categories were being reported as success and as failures. The results of this analysis are shown in Figure 3

Again, it appears that individual categories are just as likely to be viewed, within a given project, as a contributor to success as to failure.

In an effort to determine whether these strong correlations are related to the categorization process or are inherent within the data, we are currently performing a more detailed analysis of these reports.

\section{Examples From Real Games}

The initial results from our analysis of the Postmortem columns led us to conclude that weak management of the transition from preproduction to production was a source of many issues. We now look at some examples from real games that have either been published or are currently in developmen ${ }^{2}$ to find further support for this conclusion. We look at 3 issues in particular: documentation transformation, implication creating emergent requirements, and the effects of a priori knowledge on the requirements engineering process.

\subsection{Documentation Transformation}

Table 1 illustrates a microcosm of the documentation transformation issue. The game designer begins (1) with a story written in a narrative style. That story is then translated (elsewhere in the game design document) to a more formal form (2) that describes the action as a task and a justification for that task. The requirements engineer analyzes

\footnotetext{
${ }^{2}$ In the first example, minor changes have been made to the material to obfuscate the source.
}

this information, in context, to determine a set of requirements (3): identifying in-game assets such as the player avatar, Anna (a Non-Player Character (NPC)) and an inventory item. A state that controls the player's progress through the game is also identified and captured. Depending on the in-house process used, the detailed description (4) of these in-game assets may be part of the requirements document or part of a specification document. Independent of where the detailed descriptions are located, they could easily reach 50 pages once issues like artistic style, animation, and game state are included.

Performing and managing this transformation is complex. Each of these documents requires a different writing style and a single individual may not have the requisite writing skills to author materials for all purposes. In addition, creating the requirements document or specification document often requires considerable a priori knowledge of the available technology so that this information can be presented in context. There is also a multiplicative effect: each successive document is larger than the prior document as the author(s) attempt to precisely capture the required information. The authors must manage multiple stakeholder viewpoints, synthesizing a common domain language, numerous nonfunctional requirements, and inconsistencies as the project evolves.

The list of required skills is long and implies a team effort. The associated costs are significant, leading to a strong management bias toward minimizing the documentation effort.

\subsection{Implication}

By its nature as a creative work, a game design document is replete with implied information. Identifying these implications requires careful analysis, understanding the ramifications of the implications requires significant domain knowledge.

To expand on the importance of domain knowledge, we revisit Table 1. This table captures what we call first-level implications: those implications that can be derived directly from the materials presented. Almost all development teams, independent of their experience levels, capture these implications. Missing implications at this level is usually an oversight on the part of the team.

The second level of implication requires general knowledge of the domain - in this case, the adventure game genre. These implications are generally captured by teams with members who have experience with non-trivial software development projects in the domain. In this case, the description contains significant implications regarding the game world: the characters must be situated within the appropriate environment(s). Therefore, there is an environment surrounding the player when they receive the infor- 


\begin{tabular}{|c|l|l|}
\hline 1 & Story & $\begin{array}{l}\text { After her father, Bernard, died, Crystal did not know which way to turn - paralyzed by her loss } \\
\text { until the fateful day when his Will was read. }\end{array}$ \\
\hline 2 & Game Design Document & $\begin{array}{l}\text { The Player must visit Anna the Lawyer to receive a copy of Bernard's Last Will and Testament, } \\
\text { thereby obtaining the information necessary to progress to the next goal. }\end{array}$ \\
\hline 3 & Requirements & $\begin{array}{l}\text { The Player must be represented by an avatar. } \\
\text { Female Non Player Character required: Anna the Lawyer } \\
\text { Inventory Item: Last Will and Testament (LWT) } \\
\text { Player can not progress beyond Game State XYZ until LWT added to Inventory }\end{array}$ \\
\hline 4 & Specification & Could easily reach 50 pages \\
\hline
\end{tabular}

Table 1. Documentation Transformation

mation, there is Anna's office, perhaps an office building with other office interiors, background sounds, and possibly even other NPCs in the office areas. And, if there are other NPCs, do the NPCs interact with the Player?

These second level implications could easily amount to many person-months of development effort by modelers, artists, animators, and other members of the production team.

The third level of implication requires knowledge of implementation details such as the target architecture. These implications are captured by experienced teams, particularly when the present project is a sequel of some form. The requirement for the player to visit Anna raises questions about the connectivity between the elements (locales) of the virtual world - is there more than one way the Player can get to Anna the Lawyer? How does the player experience the journey? Via a scene change? Or, must they guide their avatar through the virtual world (implying the creation of all the media assets to represent the world)?

Perhaps more importantly, does the connectivity change over time? Dynamic connectivity has significant implications for representing game state (the current state of the world simulation). Designing, verifying, and maintaining a stateful world is more complex than a stateless world.

A question is raised by identifying these three levels of implication: Is it more appropriate to follow a traditional iterative process and allow these issues to surface later, or should this feedback be applied as early as possible in the process? Intuitively, early feedback is better. However, early feedback could have a negative effect on the creative process: if the game design team feels that the production team is going to reject their proposals then they may become conditioned to be less creative. The effects of early production feedback on the preproduction process merits further investigation.

\subsection{A Priori Knowledge}

Building on the analysis of the prior section, we now look more closely at the effect of a priori knowledge on the requirements engineering process.

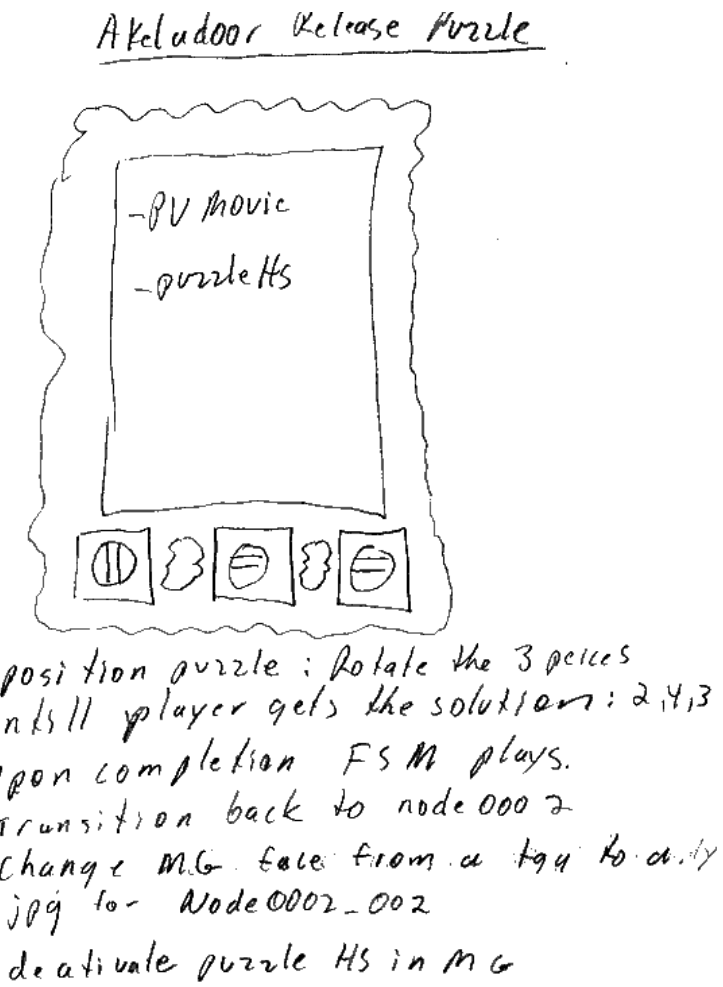

Figure 4. Akeladoor Puzzle Description, used with permission

Domain specific terms, particularly abbreviations and acronyms, are common in working papers. Figure 4 is the game designer's description of the Akeladoor Release Puzzle from the game Apocalypse Spell, currently under development by Far Vista Studios. Upon inspection, we see PV Movie: Partial Video, a less than full screen video clip, puzzle $H S$ : a puzzle Hot Spot, an interaction point for the player, FSM: Finite State Machine, $M G$ : Master Guidelines (the game uses a model driven architecture whose repository is called the Master Guidelines by the team).

If one attempts to formalize this document, they must 
understand large portions of both the preproduction and production realms. In a typical studio, this implies senior personnel from the preproduction or production staff but they are usually "too busy" to perform the task. Documentation is often assigned to a junior staff member with the rationalization that this task will "bring them up to speed".

Another alternative is to add professional technical writing resources to the projects. However, there is often a perception that it takes more time to explain it to the technical writer than it does to just write it oneself. Once this excuse is in place, no writer is hired, and soon, little or no documentation is maintained.

Significant elements of the game design documentation are informal, often with significant visual content. Visual content is particularly difficult to represent in a formal manner: iterations are often sketched as shown in the Pyramid Puzzle description of Figure 5 Careful examination of this Figure reveals evidence of prior iterations that were simply erased. Maintaining an iteration history of sketches, such as this working paper, is challenging. An electronic form of the working paper may have captured the revisions, but probably would not have captured the justifications for making the changes - often an important piece of information later in the development cycle. This information could lead to evolutionary changes in the game engine, perhaps even to a product family architecture.

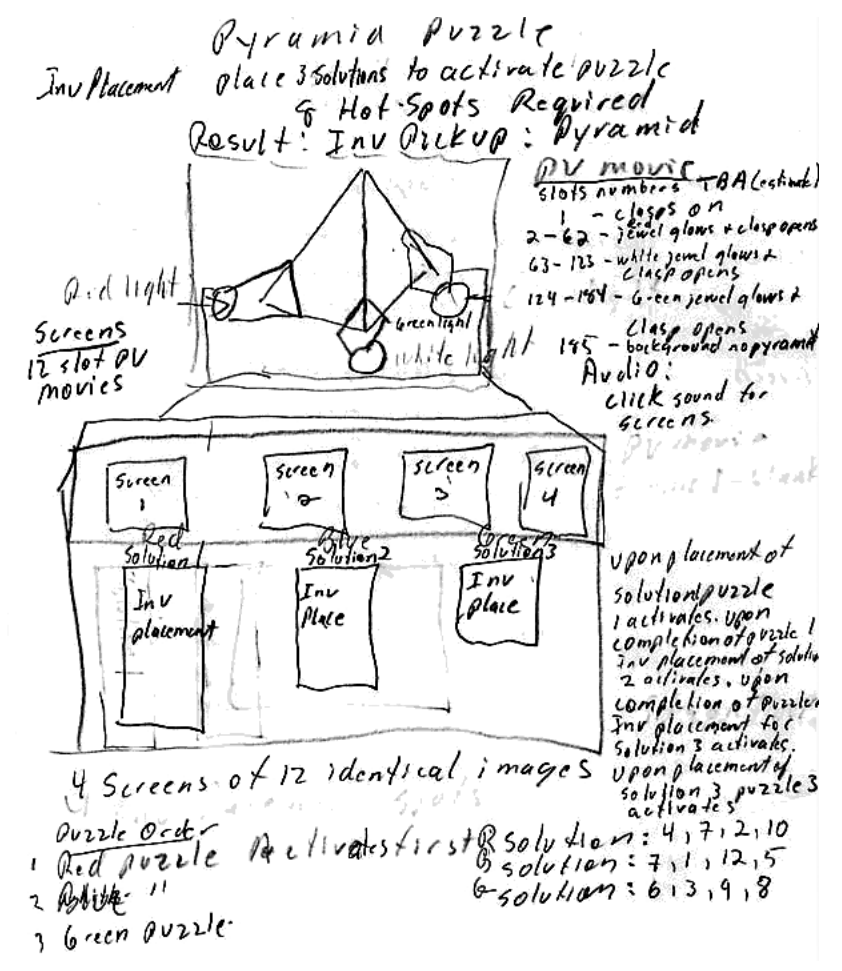

Figure 5. Pyramid Puzzle Description, used with permission
A detailed explanation of the puzzle is beyond the scope of this paper - suffice it to say that it is a combinational puzzle that requires the player to generate the correct sequence of symbols on the screens below the pyramid, one sequence for each corner of the base of the pyramid. However, application of domain knowledge during the requirements capture phase led to significant changes in the design of the puzzle.

The first issue was puzzle complexity. Solution hints were provided in the form of inventory items that looked like papyrus scrolls but there was no way for the player to show the scroll and the puzzle at the same time - the game engine simply did not support simultaneous operation of inventory inspection and puzzle modes.

The game designer was informed of this restriction and it was suggested that a place be made on the puzzle for the player to "hang" the scrolls so that they could see them while playing the puzzle. The result of this feedback was the layout of Figure 5 where the scrolls for each corner had a specific location.

This new layout raised an issue of screen resolution. The puzzle design called for an upper region for special effects, a middle region for puzzle input, and a lower region for puzzle solution hints. Unfortunately, this layout was beyond the resolution of the target platform so an alternative layout was required.

The final layout, shown in Figure 6, is a compromise between the game designer's vision, the technical capabilities of the game engine, and the technology constraints of the target platform. Only one hints scroll is visible at a time, requiring the player to shift between inventory and puzzle modes for each corner of the pyramid - not an ideal solution from a human factors perspective, but the best that could be achieved within the constraints.

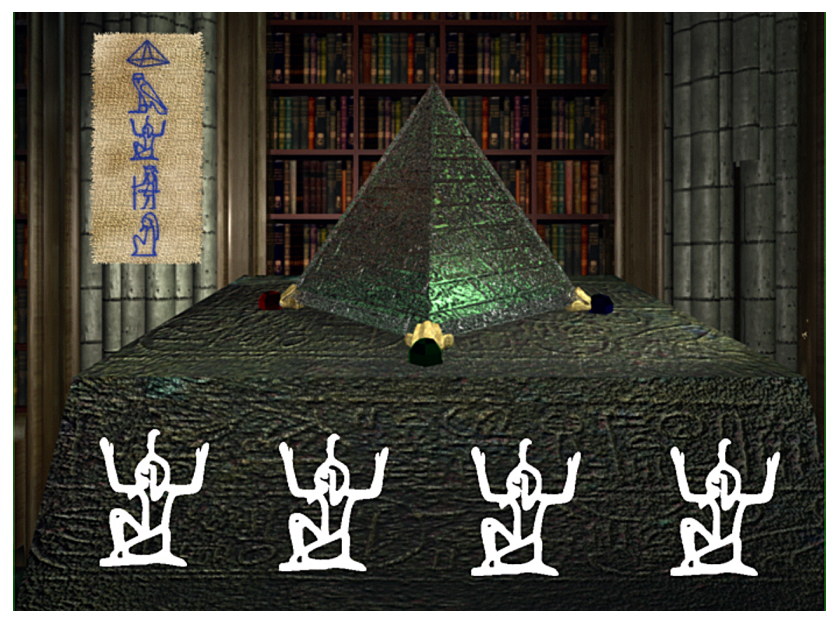

Figure 6. Pyramid puzzle prototype, used with permission 
In this example, success was achieved through dialog between team members. Unfortunately, the revised requirements and specifications for the final product were never formally captured. Given that this was 1 of approximately 100 puzzles in the game, the cost of formal capture for all puzzles is significant.

The single sheet description of the puzzle resulted in the creation of the following assets: four new inventory items, 12 secondary screen elements for user interaction, three animation sequences of four seconds duration, and sound effects for user interaction and animation support. On the software side, four state machines for validating user input and three state machines for the individual corner puzzles were required. Interactions with the game world state, the current player state, inventory management, and the save game subsystem also had to be managed. None of these assets were explicitly identified by the designer; rather, they were implied in the description of the puzzle. It can be argued that identifying these implied assets if a function of the design process. However, accurately predicting the magnitude of the production effort requires their identification at the earliest possible stage in the process.

Given that this was just was one of approximately 100 puzzles in the game, it is highly desirable that the process for identifying the implied assets and side-effects be efficient. However, we are unaware of any work in this area.

\section{Summary and Conclusions}

We have analyzed the video game development process from the perspective of requirements engineering, presented a model for video game development that integrates preproduction with production, and situated the game design document as an artifact of the preproduction process. Our analysis of 50 observational reports from the Postmortem column in Game Developer magazine showed that project management issues are the greatest contributors to success or failure in video game development. In the case of failure, many of these issues can be traced back to inadequate requirements engineering during the transition from preproduction to production.

Three examples from real video games provide further evidence of the importance of properly managing the transition from preproduction to production. These examples illustrate the challenges associated with transforming preproduction documents to production documents, the importance of detecting implied information as early as possible, and the effects of applying a priori knowledge from the production domain to the transition from preproduction to production.

The Pyramid Puzzle example showed that, if early versions of preproduction documentation are fed forward to the production team then the production team can provide important feedback to the preproduction team. This communication cycle enables earlier identification of emergent requirements and production constraints and may improve the reliability of the transition from preproduction to production. However, the introduction of production personnel into the preproduction process may have a chilling effect on the creativity of the preproduction team.

We show that requirements engineering practitioners can identify at least three levels of implication: 1) those implications that can be derived directly from the materials presented, 2) those implications that can only be derived with the introduction of general knowledge of the domain, and 3) those implications that can only be derived with the introduction of implementation details such as the target architecture. There is a strong relationship between experience and the ability to identify issues at each level of implication - indicating that a formal process for identifying implied information would not necessarily enable individuals with lesser experience to handle higher levels of implication without further guidance.

We postulate that the exploratory nature of attempts to capture the game design vision and the consequent the number of production iterations is due to a lack of formal process for managing the preproduction to production transition. As project complexity increases, we predict that studios will shift to more formal processes to increase the probability of success in their development efforts despite internal resistance to this formalization.

We conclude that creating documentation to support the transition from game design document through formal requirements and specifications is difficult, requiring significant preproduction and production domain knowledge to perform successfully. A formal process to support this transition would likely increase the reliability of the process.

\section{Future Work}

There is a strong relationship between the issues addressed by Lowe [17] in web-based development and with video game development issues, particularly those issues that are not technology based. This merits further investigation. We are currently performing a more detailed analysis of the observational reports from Game Developer magazine and other sources. We expect to use this information to further guide the development of a process for managing the transition between preproduction and production. A process for identifying sources of implied information, perhaps based on some form of syntactic analysis, would improve the efficiency and the accuracy of the documentation translation process. Mechanisms for capturing and stating non-functional requirements, such as fun, in a manner that can be measured and validated are also required.

Involving production personnel in the preproduction 
may lead to more efficient development or it may lead to reduced creativity. Further investigation is needed to quantify the tradeoffs.

\section{Acknowledgements}

We wish to thank Game Developer magazine for continuing the Postmortem column over the years. The first author thanks Electronic Arts for the opportunity to present an early version of this (and related) work at their British Columbia studios and for the valuable feedback he received. We also thank Richard Buckley of Far Vista Studios for access to internal game design documentation prior to release of their game.

\section{References}

[1] Consumer Electronics Association. Digital America. Published electronically at http://www.ce.org, 2003.

[2] Various Authors. Postmortem column. Game Developer, 6(5) through 11(6), May 1999 - June 2004.

[3] Todd Bentley, Lorraine Johnston, and Karola von Baggo. Putting some emotion into requirements engineering. In Proceedings of the 7th Australian Workshop on Requirements Engineering, 2002.

[4] Eric Bethke. Game Development and Production. Wordware Publishing, Inc., 2003.

[5] B. Boehm and V. Basili. Software defect reduction top 10 list. IEEE Computer, 34(1):135-137, January 2001.

[6] Karin Breitman and Julio Cesar Sampaio do Prado Leite. Ontology as a requirements engineering product. In $R e$ quirements Engineering, pages 309-319, 2003.

[7] Lawrence Chung. Non-Functional Requirements in Software Engineering. Kluwer Academic Publishers, 2000.

[8] Stephen W. Draper. Analysing fun as a candidate software requirement. Personal Technology, 3(1):1-6, 1999.

[9] Auston Grossman Editor. POSTMORTEMS from Game Developer. CMP Books, 2003.

[10] Brian Fuson. 2003 Top Boxoffice. Published electronically at http://www.hollywoodreporter.com, 2003.

[11] Vincenzo Gervasi and Bashar Nuseibeh. Lightweight validation of natural language requirements. Software Practice and Experience, 32(2):113-133, 2002.

[12] J. A. Goguen and C. Linde. Techniques for requirements elicination. In Proceedings of the International Symposium on Requirements Engineering, pages 152-164, Los Alamitos, California, 1993. IEEE CS Press.

[13] Joseph A. Goguen. The dry and the wet. In $I S C O$, pages 1-17, 1992.

[14] Marc Hassenzahl, Andreas Beu, and Michael Burmester. Engineering joy. IEEE Software, 18(1):70-76, 2001.

[15] M. Jarke, K. Pohl, R. Doemges, S. Jacobs, and H. Nissen. Requirements information management: The NATURE approach. Ingenerie des Systemes d'Informations, 2(6):609637, 1994.
[16] Francois Dominic Laramee, editor. Game Design Perspectives. Charles River Media, Inc., 2002.

[17] David Lowe. Web system requirements: an overview. Requirements Engineering, 8(2):102-113, 2003.

[18] Nenad Medvidovic and David S. Rosenblum. Domains of concern in software architectures and architecture description languages. In Proceedings of the 1997 USENIX Conference on Domain-Specific Languages, 1997.

[19] David Michael. The Indie Game Development Survival Guide. Charles River Media, Inc., 2003.

[20] Donald A. Norman. The Design of Everyday Things. Doubleday Books by permission of Basic Books, 1988.

[21] Francisco A. C. Pinheiro. Requirements honesty. In International Workshop on Time-Constrained Requirements Engineering (TCRE'02), Essen, Germany, September 2002.

[22] Suzane Robertson. Requirements Trawling: techniques for discovering requirements. Published electronically at http://www.systemsguild.com/GuildSite/Robs/trawling.html, 2004.

[23] Andrew Rollings and Dave Morris. Game Architecture and Design, A New Edition. New Riders Publishing, 2004.

[24] Katie Salen and Eric Zimmerman. Rules of Play: Game Design Fundamentals. MIT Press, 2004.

[25] Marc Saltzzman, editor. Game Design Secrets of the Sages. Macmillan Publishing USA, 2000.

[26] David C. Sutton. Linguistic problems with requirements and knowledge elicitation. Requirements Engineering, 5(2):114-124, 2000.

[27] Axel van Lamsweerde. Requirements engineering in the year 00: a research perspective. In International Conference on Software Engineering, pages 5-19, 2000.

[28] Pamela Zave. Classification of research efforts in requirements engineering. ACM Computing Surveys, 29(4):315321, 1997. 